\title{
Eberspächer, Achim: Das Projekt Futurologie. Über Zukunft und Fortschritt in der Bundesrepublik 1952-1982, 412 S., Schöningh, Paderborn u. a. 2018.
}

\author{
Joachim Radkau \\ Online publiziert: 17. Januar 2020 \\ (C) Der/die Autor(en) 2019
}

Das Buch beginnt mit dem „Spiegel“-Titel vom Jahresende 1966, mit dem - wie der Verfasser Achim Eberspächer wiederholt hervorhebt (S. 174, 341) - die bundesdeutsche „Medienkarriere“ der Futurologie begann. Unten der Titel „Futurologie. Die Zukunft des Menschen wird geplant“, darüber ein Gewirr von Antennen, worunter man sich eine riesige Sendestation vorstellen mag. Der Titel hat es in sich, und dies in mehrfacher Hinsicht. Er setzt Zukunftsforschung mit Zukunftsplanung gleich; und in der Tat suchten sich ambitiöse Futurologen den Politikern als Planer anzudienen.

Eberspächer, Jahrgang 1980, ist wissenschaftlicher Referent für Energiesysteme der Zukunft an der Deutschen Akademie der Technikwissenschaften (acatech), ist also in gewissem Sinne von Beruf selber Futurologe, obwohl dieser Terminus, wie er immer wieder zeigt, längst in Verruf geraten ist; sein Buch ist als Dissertation entstanden. Er verhehlt nicht, dass es zu seinem Thema bereits ein anderes umfangreiches neues Opus gibt: das aus einer Habilitationsschrift hervorgegangene Buch von Elke Seefried: „Zukünfte. Aufstieg und Krise der Zukunftsforschung 1945-1980“ (2015). Wenn er bemerkt (S. 31), Seefried habe ihre Arbeit nach der seinigen begonnen, jedoch vor ihr publiziert, spürt man zwischen den Zeilen den Verdruss. Da waren weite inhaltliche Überschneidungen schwer zu vermeiden; zugleich erkennt man jedoch in Eberspächers Buch durchgängig ein Bestreben, gegenüber Seefried ein eigenes Profil zu gewinnen.

Daher hat es seinen Reiz, beide Bücher nebeneinander zu lesen, wobei sich allerdings zugleich ein widersprüchliches, ja heillos verwirrendes Bild von der Futurologie und ihrer Geschichte ergibt. Ein besonders krasses Beispiel: Herman Kahn, der in den USA ,als der ungekrönte König unter den Futurologen“ galt (Claus Grossner bei Eberspächer, S. 302) und 1970 bei einem Vortrag in Frankfurt „stolze 650 DM Eintrittsgeld" kassierte (S. 303), wird von Seefried (S. 111) mit seinem Kalkül zitiert, „es sei besser, man verliere 40 Millionen Amerikaner in einem Nuklearkrieg und der Rest könne in Freiheit leben“. Eberspächer dagegen (S. 304) weist darauf hin, dass dieser wendige Koloss von Mann von Robert Jungk 1967 ,öffentlich in

J. Radkau ( $\bowtie)$

Bielefeld, Deutschland

E-Mail: joachim.radkau@uni-bielefeld.de 
Schutz genommen“" worden sei: von Jungk, der sich der Friedensforschung genähert hatte!

Nicht wenige Leser mögen bei der Lektüre immer ungeduldiger darauf warten, was denn nun diese Futurologen konkret prophezeit haben; doch da lassen sie Eberspächer wie Seefried zappeln, und das hat seinen Grund. Jene Zukunftsforscher, die professionell werden wollten, waren nämlich in der Regel mit konkreten Aussagen zurückhaltend: Sie wollten ja eben vor Augen führen, dass man, um zu fundierten Prognosen zu gelangen, erst einmal entsprechende Institute und Organisationen begründen und dafür Fördermittel erlangen müsse.

Den Startschuss zur späteren Futurologie gab 1952 Robert Jungk mit seinem Bestseller „Die Zukunft hat schon begonnen“, der - so Eberspächer (S. 45) - ,wie eine Bombe“ einschlug. Der Titel, auf den der Autor erst durch seinen Verleger gebracht worden war - Jungk selbst hatte das Buch ursprünglich einfach „Amerika 1952“ nennen wollen (S. 39) - avancierte rasch zum geflügelten Wort. Doch was war das für eine Zukunft? Sie lag in den USA, und zwar in den amerikanischen Denkfabriken, die vielfach mit dem Militär verbunden waren; was sie im Laufe der Zeit ausbrüten würden, war die große Frage.

Im Zentrum dessen, was später in der Bundesrepublik als Futurologie lief, standen in Eberspächers Darstellung eher ethische Forderungen im Dienste der Friedenserhaltung und Sicherung der menschlichen Existenz; in den konkreten Prognosen ergingen sich eher die Medien - und doch wirkten diese manchmal auf die Futurologie zurück, was dazu beitrug, diese in Misskredit zu bringen. Besonders skrupellos war in dieser Hinsicht Herman Kahn im Verbund mit seinem futurologischen Kollegen Anthony Wiener: mit dem Buch „The Year 2000“ (1967), das 1968 auf Deutsch unter dem Titel „Ihr werdet es erleben“ herauskam, für die Öffentlichkeit zum Inbegriff der Futurologie wurde und deutsche Publikationen in den Schatten stellte (S. 199f.). Es enthält so viele Fehlprognosen, dass es vielleicht Kahns Glück war, dass er die Jahrtausendwende nicht mehr erlebte: Schon 1983 starb er mit nur 61 Jahren an einem Herzinfarkt.

Im Unterschied zu Seefried, die auch US-amerikanische und französische Ursprünge, überhaupt die internationale Ebene der Futurologie ausführlich behandelt, beschränkt sich Eberspächer, wenn auch mit vielen transnationalen Seitenblicken, auf die Bundesrepublik Deutschland, bietet dafür dort eine Fülle an aufschlussreichen Details, wobei seine in eher erzählendem Stil geschriebene Darstellung sich für eine Dissertation durch gute Lesbarkeit auszeichnet. Der heutige Zukunftshistoriker, der sein Metier für neuartig hält, liest mit einiger Verblüffung (S. $91 \mathrm{f}$.), dass 1962 in Duisburg ein Historikertag zum Thema „Zukunft in der Geschichte“ stattfand umso merkwürdiger, dass dennoch Ansätze zur Zukunftsgeschichte immer wieder stecken blieben! Schon 1956 hatte der Direktor von Ruhrgas, Fritz Gummert, einen Lehrstuhl „Geschichte der Zukunft“ gefordert (S. 82).

In alter, heute manchmal gar zu sehr vernachlässigter Historikertradition handelt Eberspächers Untersuchung viel von Menschen, nicht in gleichem Maße von überindividuellen Trends und Strukturen; wie er vorausschickt (S. 10), schreibt er die Geschichte der Futurologie ,entlang der drei Hauptdarsteller Flechtheim, Jungk und Steinbuch", wobei diese drei freilich oftmals aus dem Blick geraten; nur begrenzt lässt sich die Geschichte der Zukunftsspekulationen an bestimmten Personen fest- 
machen. Ob Ossip Flechtheim (1909-1998) tatsächlich zu den „Hauptdarstellern“ gehört, darüber lässt sich streiten: Zwar erfand er 1943 im US-Exil den Terminus „futurology“, doch jahrzehntelang griff ihn kaum jemand auf, und im Futurologie-Boom der späten 1960er Jahre bleibt er eher eine etwas blasse Randfigur. Die wortmächtigsten Exponenten waren Robert Jungk (1913-1994) und Karl Steinbuch (1917-2005). Eberspächer hat deren Nachlässe durchstöbert, was seiner Darstellung streckenweise den Reiz des Intimen gibt; so fand er eine Aussage, dass Jungk, der 1968/69 an der Berliner Technischen Universität im neuen antiautoritären Stil ein Seminar über „Möglichkeiten der technologischen Voraussage“ abhielt, „fast wie ein Heiliger von den Studenten behandelt worden“ sei (S. 220), wogegen Steinbuch, als er von links auf scharf antilinks umgeschwenkt war, als „Nazischwein“ (S. 249) und ,Drecksack“ (S. 316) beschimpft wurde.

Bei Eberspächer wie schon bei Seefried erkennt man, dass die Geschichte der Futurologie ihre dramatischen Seiten hat. Sie entstand, wie Seefried darstellt - was Eberspächer mit seiner deutschen Sicht in Frage zu stellen sucht - in den USA als Cold War Science, ging dagegen in der Bundesrepublik teilweise ein Bündnis mit der Friedensforschung ein. Der Fortgang der Futurologie beschreibt ein markantes Auf und Ab, mit dem Höhepunkt im Jahre 1968 (S. 196): Da liegt die Frage nahe, ob die ,Achtundsechziger' von der Futurologie inspiriert wurden; doch auf diese Frage gibt es keine einfache Antwort. Hans Magnus Enzensbergers Kursbuch erschien 1968 unter dem Titel „Kritik der Zukunft“ und begann mit einer Attacke auf die Futurologie (S. 208 ff.); weiter hinten jedoch wie auch anderswo zeigten sich führende Achtundsechziger durchaus aufnahmebereit für visionäre Erwartungen vom „Fortschritt der Produktivkräfte“.

Schon ab 1969 galt die Futurologie in der Wissenschaft weithin als unseriös, wenn nicht geradezu als Ausdruck von Größenwahn; doch damit war das Zukunftsdenken, das nach wissenschaftlicher Basis sucht, keineswegs am Ende. Vielmehr wurden „Die Grenzen des Wachstums“ - darin stimmen Eberspächer (S. 285 ff.) und Seefried überein - in der Prognostik epochal; in welchem Maße die neue Umweltbewegung von der Sorge um die Zukunft getragen wurde - mit dem Klima-Alarm als bisheriger Klimax -, bleibt allerdings in beiden Darstellungen eher am Rande. Ein persönliches Drama dagegen, in das Eberspächer delikate Einblicke liefert, ist der Wandel der Beziehung zwischen Jungk und Steinbuch: Die anfängliche Kampfgemeinschaft schlug ziemlich abrupt in offene Feindschaft um, wobei Steinbuch, der sich zum Wüterich wandelte, gegen den einstigen Mitstreiter eine förmliche „Schlammschlacht“ führte (S. 250, 343) und - man staune! - schließlich die gesamte Futurologie, als deren aufsteigender Führer er sich gebärdet hatte, als „Unsinn“ abkanzelte (S. 317)! In den 1970er Jahren stellte Jungk sich mit seinem „Atomstaat“ (1977) an die Spitze der Anti-AKW-Bewegung, wogegen Steinbuch seinem einstigen Mitstreiter „Hass auf wissenschaftlichen Sachverstand“, „halluzinogene Futurologie“ (S. 337) und ,primitives Maschinenstürmertum“ (S. 244) vorwarf und gegen vermeintliche ,Technikfeinde“ wütete - keine Idee davon, dass gerade die Umweltbewegung im Endeffekt neue ,,alternative“ Technologien vorantrieb!

Eberspächer schickt seiner Darstellung voraus (S. 9f.; vgl. auch S. $62 \mathrm{ff}$.), dass seine „drei Hauptdarsteller“ sich in einem einig gewesen seien: ,dass der naturwissenschaftlich-technische Fortschritt diese neue Epoche dominieren und dabei 
zahlreiche ethische Fragen und praktische Herausforderungen aufwerfen würde." Allgemein wurde es gängig, nicht mehr von neuen Techniken, sondern von „Technologien“ zu reden; der Begriff suggerierte, diese Techniken besäßen ihre eigene Entwicklungslogik. Eberspächer behandelt vor allem Raumfahrt und Kernenergie als „wirkmächtige Technikvisionen“ (S. 62 ff.); doch auch die Computer spielten in der Futurologie eine Schlüsselrolle, zumal man in den 1960er Jahren glaubte, diese würden immer größer und teurer werden und daher Instrumente der Macht sein noch keine Ahnung davon, dass in einigen Jahrzehnten fast jedes Kind sein Smartphone haben würde! Doch schon das Nebeneinander von militärischer und ziviler Kerntechnik war eigentlich der schlagende Beweis, dass die ,Technologie“ nicht in sich ihre Logik besitzt! Dass es nicht die vorhersehbare Zukunft gibt, sondern mehrere mögliche Zukünfte, wurde denn auch von Futurologen im Prinzip immer wieder anerkannt, doch nicht konsequent beachtet. Medienwirksam waren plurale Zukünfte nach dem Muster Paradies/Apokalypse.

Wann und woran scheiterte die Futurologie der fünfziger und sechziger Jahre? Den entscheidenden Grund erkennt Eberspächer abschließend darin, dass es diesen Zukunftsforschern nicht gelungen sei, ,die von den Auftraggebern gefragte umsetzbare Expertise zu liefern“" (S. 338). Die Schilderung, wie die Zusammenarbeit der Heidelberger Zukunftsforscher unter Helmut Krauch mit dem ehrgeizigen Kanzleramts-Staatssekretär Horst Ehmke für die Futurologen zur Quälerei wurde, war bereits ein Highlight bei Seefried gewesen; vermutlich deshalb kommt diese Geschichte bei Eberspächer nur am Rande vor. Er zieht das „Fazit“ (S. 343), die „öffentliche Schlammschlacht“ zwischen Steinbuch und Jungk habe „das Ansehen und die Handlungsfähigkeit der futurologischen Institutionen schwer beschädigt". Sie hätten soviel „Zeit und Energie“ in ,den Streit untereinander“ investiert, dass sie zur produktiven Weiterentwicklung der Futurologie nicht gekommen seien (S. 273). Je nach Standpunkt kann man diese Geschichten als Tragödie oder Komödie betrachten. Der politisch erfahrene Philosoph Hermann Lübbe, auf den sich Eberspächer anders als Seefried häufig beruft, kritisierte 1969 die Futurologie unter dem Titel: „Ernst und Unernst der Zukunftsforschung“ (S. 214f.).

Ein tieferer Grund des Scheiterns lässt sich jedoch in weiten Teilen der Darstellung erkennen: dass eine Futurologie als solide Wissenschaft prinzipiell unmöglich ist, und dies gerade dann, wenn man in dem wissenschaftlich-technischen Fortschritt den entscheidenden Faktor der Zukunft erblickt. Wie Eberspächer an früherer Stelle hervorhebt (S. 213), hatte der Wissenschaftstheoretiker Karl Popper schon Anfang der 1930er Jahre das entscheidende Argument mit dem später so genannten „PopperTheorem“ „auf den Punkt gebracht“, um ihn selbst zu zitieren: „wenn es so etwas wie ein wachsendes menschliches Wissen gibt, dann können wir nicht heute das vorwegnehmen, was wir erst morgen wissen werden.“

Funding Open Access funding provided by Projekt DEAL.

Weitere Details zur Lizenz entnehmen Sie bitte der Lizenzinformation auf http://creativecommons.org/ licenses/by/4.0/deed.de.

Die in diesem Artikel enthaltenen Bilder und sonstiges Drittmaterial unterliegen ebenfalls der genannten Creative Commons Lizenz, sofern sich aus der Abbildungslegende nichts anderes ergibt. Sofern das betreffende Material nicht unter der genannten Creative Commons Lizenz steht und die betreffende Handlung 
nicht nach gesetzlichen Vorschriften erlaubt ist, ist für die oben aufgeführten Weiterverwendungen des Materials die Einwilligung des jeweiligen Rechteinhabers einzuholen.

Open Access Dieser Artikel wird unter der Creative Commons Namensnennung 4.0 International Lizenz veröffentlicht, welche die Nutzung, Vervielfältigung, Bearbeitung, Verbreitung und Wiedergabe in jeglichem Medium und Format erlaubt, sofern Sie den/die ursprünglichen Autor(en) und die Quelle ordnungsgemäß nennen, einen Link zur Creative Commons Lizenz beifügen und angeben, ob Änderungen vorgenommen wurden.

\title{
Mrozek, Bodo: Jugend - Pop - Kultur. Eine transnationale Geschichte, 866 S., Suhrkamp, Berlin 2019.
}

\author{
Barbara Stambolis \\ Online publiziert: 10. Dezember 2019 \\ (C) Gesellschaft zur wissenschaftlichen Förderung politischer Literatur e.V. and the Author(s) 2019
}

Der Historiker und Journalist Bodo Mrozek, Mitarbeiter am Zentrum für Zeithistorische Forschung, der seit Sommersemester 2018 die Professur für Theorie und Geschichte der populären Musik der Humboldt-Universität zu Berlin vertritt, wurde mit der vorliegenden, für die Veröffentlichung leicht veränderten Arbeit im Jahre 2016 promoviert. Allerdings handelt es sich um weit mehr als eine Dissertation: Der umfangreichen Studie liegen langjährige Forschungen und auch bereits einschlägige Publikationen zugrunde, in denen Mrozek sich intensiv mit Fragen der Begrifflichkeit, methodischen Ansätzen und einzelnen Aspekten der weiten Thematik befasst hat, und zwar im Austausch mit Kolleginnen und Kollegen aus unterschiedlichen Wissenschaftsdisziplinen. Zudem verfügt der Autor über eine fundierte Kenntnis aktueller Forschungstendenzen sowie einen breiten, für Zeithistoriker nicht selbstverständlichen Quellenfundus. Um es bereits an dieser Stelle deutlich festzuhalten: Die Studie überzeugt nicht nur durch die Breite des Themenfeldes, sondern auch durch die souveräne Verknüpfung unterschiedlicher methodischer Zugänge, der vielfältige wissenschaftliche, enge Fachgrenzen der Zeitgeschichtsforschung überschreitende Vernetzungen zugrunde liegen.

In der Einleitung werden die im Titel der Untersuchung angesprochenen Forschungsfelder, Begriffe, Leitfragen, Quellen und andere umrissen. Mrozek betont,

B. Stambolis $(\bowtie)$

Münster, Deutschland

E-Mail: barbarastambolis@outlook.de 